\title{
96. Infinitely Many Periodic Solutions for a Superlinear Forced Wave Equation
}

\author{
By Kazunaga TANAKA \\ Department of Mathematics, Waseda University \\ (Communicated by Kôsaku Yosida, M. J. A., Dec. 12, 1985)
}

1. Introduction. In this article we shall study the nonlinear wave equation :

$$
\begin{array}{ll}
v_{t t}-v_{x x}+g(v)=f(x, t), & (x, t) \in(0, \pi) \times \boldsymbol{R}, \\
v(0, t)=v(\pi, t)=0, & t \in \boldsymbol{R}, \\
v(x, t+2 \pi)=v(x, t), & (x, t) \in(0, \pi) \times \boldsymbol{R},
\end{array}
$$

where $g \in C(\boldsymbol{R}, \boldsymbol{R})$ is a function such that $g(\xi) / \xi \rightarrow \infty$ as $|\xi| \rightarrow \infty$ and $f(x, t)$ is a $2 \pi$-periodic function of $t$.

In a previous paper K. Tanaka [5] we studied (1)-(3) in case $g(\xi)$ $= \pm|\xi|^{s-1} \xi$. This paper is a continuation of [5] and deals with more general equations. Our main result is as follows :

Theorem. Suppose that $g \in C(R, R)$ satisfies

$\left(\mathrm{g}_{1}\right) \quad g(\xi)$ is strictly increasing,

$\left(\mathrm{g}_{2}\right)$ there exist $\mu>2$ and $l \geqq 0$ such that for $|\xi| \geqq l$,

$$
0<\mu G(\xi) \equiv \mu \int_{0}^{\xi} g(\tau) d \tau \leqq \xi g(\xi),
$$

$\left(\mathrm{g}_{3}\right)$ there exist $s>1$ and $C>0$ such that for $\xi \in R$,

$$
|g(\xi)| \leqq C\left(|\xi|^{s}+1\right)
$$

$\left(\mathrm{g}_{4}\right) \frac{2}{s-1}>\frac{\mu}{\mu-1}$.

Then, for all $2 \pi$-periodic $f(x, t) \in L^{\infty}([0, \pi] \times R)$, there exists an unbounded sequence of weak solutions of (1)-(3) in $L^{\infty}$.

In [3], P. H. Rabinowitz obtained the conditions which ensure the existence of an unbounded sequence of solutions of the semilinear elliptic equation :

$$
\begin{aligned}
-\Delta u & =g(u)+f(x), & & x \in D, \\
u & =0, & & x \in \partial D,
\end{aligned}
$$

where $D \subset \boldsymbol{R}^{n}$ is a smooth bounded domain. In particular, in case $n=2$, his conditions are $\left(\mathrm{g}_{2}\right),\left(\mathrm{g}_{3}\right),\left(\mathrm{g}_{4}\right)$ and

$$
\left(\mathrm{g}_{5}\right) \quad g(-\xi)=-g(\xi) \quad \text { for all } \xi \in R .
$$

He also obtained a similar existence result for the second order Hamiltonian systems of ordinary differential equations. For the wave equation (1)-(3), we act on $S^{1}$-symmetry and get the existence result without assumption $\left(\mathrm{g}_{5}\right)$.

As in K. Tanaka [5], we use a perturbation result of P. H. Rabinowitz [3] asserting the existence of infinitely many critical points of perturbed 
symmetric functionals and the dual variational formulation of the problem (1)-(3). Details of the proof will be published elsewhere.

2. Outline of the proof. Let $\Omega=(0, \pi) \times(0,2 \pi)$ and $h(\xi)=$ the inverse function of $g(\xi)$. Set

$$
q=\frac{\mu}{\mu-1} \in(1,2) \text { and } \quad r=\frac{1}{s}+1 .
$$

Consider the operator $A u=u_{t t}-u_{x x}$ acting on functions in $L^{1}(\Omega)$ satisfying (2) and (3). Denote by $N$ the kernel of $A$. We act on the space

$$
E=\left\{u \in L^{q}(\Omega) ; \int_{\Omega} u \phi=0 \text { for all } \phi \in N \cap L^{\mu}(\Omega)\right\}
$$

with $L^{q}$ norm $\|\cdot\|_{q}$. For $\theta \in[0,2 \pi) \simeq S^{1}$, define $T_{\theta}: E \rightarrow E$ by $\left(T_{\theta} u\right)(x, t)$ $=u(x, t+\theta)$.

For any $u \in E$ there exists a unique $K u \in E$ such that $A(K u)=u$. Moreover the operator $K: E \rightarrow E^{*}$ is compact.

We define the functional $I(u) \in C^{1}(E, R)$ by

$$
I(u)=\frac{1}{2} \int_{\Omega}(K u) u+\int_{\Omega} H(u+f),
$$

where $H(\xi)=\int_{0}^{\xi} h(\tau) d \tau$. There is a one-to-one correspondence between the critical points of $I(u)$ and the weak solutions of (1)-(3).

To verify the Palais-Smale compactness condition, we replace $I(u)$ by $I(\varepsilon ; u) \in C^{1}(E, R)(\varepsilon \in[0,1])$ defined by

$$
I(\varepsilon ; u)=\frac{1}{2} \int_{\Omega}(K u) u+\int_{\Omega} H(u+f)+\int_{\Omega} \omega(\varepsilon u),
$$

where $\omega \in C^{2}(R, R)$ is an even convex function such that $\omega(\xi)=|\xi|^{q}$ for $|\xi| \geqq 1$, $\omega(\xi)=0$ for $|\xi| \leqq c_{q}$, where $c_{q}>0$ is a constant. Then $I(\varepsilon ; u)$ satisfy the Palais-Smale condition for all $\varepsilon \in(0,1]$.

As in K. Tanaka [5], we use another modified functional $J(\varepsilon ; u)$ $\in C^{1}(E, R)$ defined by

$$
J(\varepsilon ; u)=\frac{1}{2} \int_{\Omega}(K u) u+\int_{\Omega} H(u)+\int_{\Omega} \omega(\varepsilon u)+\psi(\varepsilon ; u) \int_{\Omega}(H(u+f)-H(u)),
$$

where $\psi(\varepsilon ; u)$ will be defined analogously as in $\mathrm{K}$. Tanaka [5]. Here we can assume that $J(\varepsilon ; u)$ is a nondecreasing function of $\varepsilon \in[0,1]$ for fixed $u \in E$. In what follows we denote by " " the Fréchet derivative with respect to $u$.

Lemma 1. There is a constant $M>0$ independent of $\varepsilon \in(0,1]$ such that

(i) $J(\varepsilon ; u)$ satisfies the Palais-Smale condition on

$$
\hat{A}_{M}(\varepsilon)=\{u \in E ; J(\varepsilon ; u) \geqq M\} \text {. }
$$

(ii) $J(\varepsilon ; u) \geqq M$ and $J^{\prime}(\varepsilon ; u)=0$ imply that $J(\varepsilon ; u)=I(\varepsilon ; u)$ and $I^{\prime}(\varepsilon ; u)$ $=0$.

Note that $K$ is a compact self-adjoint operator in $E \cap L^{2}(\Omega)$. Its eigenvalues are $\left\{1 /\left(j^{2}-k^{2}\right) ; j \neq k\right\}$. We rearrange the negative eigenvalues in the following order, denoted by

$$
-\mu_{1} \leqq-\mu_{2} \leqq-\mu_{3} \leqq \cdots<0 \text {. }
$$


Here, for each $n$, there is a one-to-one correspondence between $\mu_{n}$ and a 2-dimensional invariant subspace :

Define

$$
\operatorname{Span}\left\{e_{n}^{+}=\sin j x \cdot \cos k t, e_{n}^{-}=\sin j x \cdot \sin k t\right\} \quad\left(j^{2}-k^{2}=-\mu_{n}^{-1}\right) .
$$

$$
E_{n}=\operatorname{span}\left\{e_{1}^{+}, e_{1}^{-}, e_{2}^{+}, e_{2}^{-}, \cdots, e_{n}^{+}, e_{n}^{-}\right\} .
$$

Clearly there exists a sequence of numbers : $0<R_{1}<R_{2}<\cdots$ such that

$$
\begin{aligned}
J(\varepsilon ; u) \leqq 0 & \text { for all } u \in E_{n} \quad \text { with }\|u\|_{r} \geqq R_{n} \\
& \text { and for all } \varepsilon \in[0,1] .
\end{aligned}
$$

Let

$$
B_{R}=\left\{u \in E ;\|u\|_{r} \leqq R\right\}, \quad D_{n}=B_{R_{n}} \cap E_{n},
$$

$\Gamma_{n}=\left\{\gamma \in C\left(D_{n}, E\right) ; \gamma\left(T_{\theta} u\right)=T_{\theta} \gamma(u)\right.$ for all $u$ and $\theta, \gamma(u)=u$ if $\left.\|u\|_{r}=R_{n}\right\}$,

$U_{n}=\left\{u=\tau e_{n+1}^{+}+w ; \tau \geqq 0, w \in B_{R_{n+1}} \cap E_{n}\right.$, and $\left.\|u\|_{r} \leqq R_{n+1}\right\}$,

$\Lambda_{n}=\left\{\lambda \in C\left(U_{n}, E\right) ;\left.\lambda\right|_{D_{n}} \in \Gamma_{n}, \lambda(u)=u\right.$ if $\|u\|_{r}=R_{n+1}$

Define for $n \in N$ and $\varepsilon \in[0,1]$,

$$
\text { or } \left.u \in\left(B_{R_{n+1}} \backslash B_{R_{n}}\right) \cap E_{n}\right\} \text {. }
$$

$$
\begin{aligned}
& b_{n}(\varepsilon)=\inf _{r \in \Gamma_{n}} \sup _{u \in D_{n}} J(\varepsilon ; \gamma(u)), \\
& c_{n}(\varepsilon)=\inf _{\lambda \in \Lambda_{n}} \sup _{u \in U_{n}} J(\varepsilon ; \lambda(u)) .
\end{aligned}
$$

The above definitions are analogous to those of P. H. Rabinowitz [3], which are used to prove the existence of solutions of the second order Hamiltonian systems.

It is clear that $c_{n}(\varepsilon) \geqq b_{n}(\varepsilon)$. In case $c_{n}(\varepsilon)>b_{n}(\varepsilon)$, as in [3], we have the following

Proposition 1. For $\varepsilon \in(0,1]$, suppose that $c_{n}(\varepsilon)>b_{n}(\varepsilon) \geqq M$. Let $d \in\left(0, c_{n}(\varepsilon)-b_{n}(\varepsilon)\right)$ and

Define

$$
\Lambda_{n}(\varepsilon ; d)=\left\{\lambda \in \Lambda_{n} ; J(\varepsilon ; \lambda(u)) \leqq b_{n}(\varepsilon)+d \text { on } D_{n}\right\} .
$$

$$
c_{n}(\varepsilon ; d)=\inf _{\lambda \in \Lambda_{n}(s ; d)} \sup _{u \in U_{n}} J(\varepsilon ; \lambda(u)) .
$$

Then, $c_{n}(\varepsilon ; d)$ is a critical value of $I(\varepsilon ; u)$.

On the other hand, as in H. Brézis, J. M. Coron and L. Nirenberg [2], we have

Proposition 2. For any $L>0$, there exists a constant $C_{L}>0$ independent of $\varepsilon \in(0,1]$ such that the assumption

imply

$$
I^{\prime}(\varepsilon ; u)=0 \text { and } I(\varepsilon ; u) \leqq L
$$

$$
\|u\|_{\infty} \leqq C_{L} .
$$

Recalling $\omega(\xi)=0$ for $|\xi| \leqq c_{q}$, for the proof of our theorem it suffices to prove the following

Proposition 3. There exists a sequence $\left\{n_{j}\right\}_{j=1}^{\infty}$ such that for some constants $\delta_{j} \in(0,1]$ and $d_{j}>0$,

$$
c_{n_{j}}(\varepsilon)-2 d_{j} \geqq b_{n_{j}}(\varepsilon) \geqq M \quad \text { for all } \varepsilon \in\left(0, \delta_{j}\right] .
$$

Moreover, there exist sequence $\left\{m_{j}\right\}_{j=1}^{\infty}$ and $\left\{M_{\}_{j}}^{\infty}\right.$ which are independent of $\varepsilon$ and 


$$
\begin{aligned}
& m_{1} \rightarrow \infty \quad \text { as } j \rightarrow \infty \text {, } \\
& m_{j} \leqq c_{n_{j}}\left(\varepsilon ; d_{j}\right) \leqq M_{j} \quad \text { for } \varepsilon \in\left(0, \delta_{j}\right] \text {. }
\end{aligned}
$$

This proposition follows from the next lemmas.

Lemma 2. There is a constant $\beta>0$ such that for $u \in E$ and $\theta \in[0,2 \pi)$, $\left|J\left(0 ; T_{\theta} u\right)-J(0 ; u)\right| \leqq \beta\left(|J(0 ; u)|^{(q-1) / q}+1\right)$.

Lemma 3. For any $\delta>0$ there is a constant $C_{\delta}>0$ such that

$$
b_{n}(0) \geqq C_{\delta} n^{2(r-1) /(2-r)-\delta} \quad \text { for all } n \in N \text {. }
$$

Lemma 4. There exists a sequence $\left\{n_{j}\right\}_{j=1}^{\infty}$ such that

$$
c_{n_{j}}(0)>b_{n_{j}}(0) \geqq M \quad \text { for all } j \in N \text {. }
$$

Lemma 5. The functions $b_{n}(\cdot), c_{n}(\cdot):[0,1] \rightarrow R$ are right-continuous. In particular, they are continuous at 0.

Here, as in $\mathrm{K}$. Tanaka [5], we derive Lemmas 2, 3, 4, from $\left(\mathrm{g}_{2}\right),\left(\mathrm{g}_{3}\right)$, $\left(\mathrm{g}_{4}\right)$ respectively. Lemma 5 is obtained from the fact that $J(\varepsilon ; u)$ is a nondecreasing function of $\varepsilon$ for fixed $u$.

Remark. It is clear that Theorem can be extended to the equation of the form :

$$
v_{t t}-v_{x x}+g(x, v)=f(x, t) \text {. }
$$

In case that $g(x, t, v)$ depends also on $t$, we must act on $Z_{2}$-symmetry as in K. Tanaka [5]. That is, we assume that $g(x, t, v)$ is odd in $v$ and satisfies similar conditions to $\left(\mathrm{g}_{1}\right)-\left(\mathrm{g}_{4}\right)$, then we have the existence result.

Acknowledgment. The author would like to thank Professor Haruo Sunouchi for his advice and encouragement.

\section{References}

[1] H. Brézis: Periodic solutions of nonlinear vibrating strings and duality principles. Bull. Amer. Math. Soc. (N.S.), 8, 409-426 (1983).

[2] H. Brézis, J. M. Coron, and L. Nirenberg: Free vibrations for a nonlinear wave equation and a theorem of P. Rabinowitz. Comm. Pure Appl. Math., 33, 667$689(1980)$.

[3] P. H. Rabinowitz: Multiple critical points of perturbed symmetric functionals. Trans. Amer. Math. Soc., 272, 753-769 (1982).

[4] - L Large amplitude time periodic solutions of a semilinear wave equation. Comm. Pure Appl. Math., 37, 189-206 (1984).

[5] K. Tanaka: Infinitely many periodic solutions for the equation: $u_{t t}-u_{x x} \pm|u|^{s-1} u$ $=f(x, t)$. Proc. Japan Acad., 61A, 70-73 (1985) (and detailed paper to appear). 